\title{
KULEUVEN
}

\section{Parametric space-frequency reduction for second-order system models}

\section{INTRODUCTION}

This research focusses on the computational cost reduction of frequency domain simulations in many-query applications with varying model parameters. These analyses are often encountered during the design of mechanical structures, where frequency response function (FRF) amplitudes are still one of the key performance metrics to be considered. Moreover, often inputs (number and frequency content) can vary broadly, which makes it all the more challenging to set up the reduced model.

For a mechanical system, the FRF matrix $\boldsymbol{H}(\boldsymbol{p}, \boldsymbol{\omega})$ for parameter set $\boldsymbol{p}$ and some input matrix $\boldsymbol{B}$ is obtained as:

$$
\boldsymbol{H}\left(\boldsymbol{p}, \omega_{i}\right)=\left(-\boldsymbol{M}\left(\boldsymbol{p}, \omega_{i}\right) \omega_{i}^{2}+j \boldsymbol{C}\left(\boldsymbol{p}, \omega_{i}\right) \omega_{i}+\boldsymbol{K}\left(\boldsymbol{p}, \omega_{i}\right)\right)^{-1} \boldsymbol{B} .
$$

The cost of evaluating this FRF for all $\omega_{i} \in \omega$ stems from:

- The large system solved at each frequency line;

The large number of frequency lines which need to be evaluated. As this is a common problem encountered in a range of disciplines, many methods have been developed to tackle this computational cost.

In this work we propose a two step approach for the rapid reduced FRF evaluation of parametric systems. This approach consists of:

- A local state projection to reduce the inversion cost;

- A frequency domain sampling and interpolation to limit the number of frequency line evaluations.

It is important to notice structural engineers are typically interested in obtaining the full motion shape at a certain frequency. This is in contrast to many other domains where the main interest is on specific $1 / O$ relations.

\section{SPACE-FREQUENCY MODEL REDUCTION}

The proposed space-frequency model order reduction approach approximates the frequency response $\widetilde{\boldsymbol{H}}\left(\boldsymbol{p}, \omega_{i}\right)$ as:

$\boldsymbol{H}\left(\boldsymbol{p}, \omega_{i}\right) \approx \widetilde{\boldsymbol{H}}\left(\boldsymbol{p}, \omega_{i}\right)$
$=\sum_{\omega_{j} \in \omega_{s}} \boldsymbol{W}_{\boldsymbol{i} \boldsymbol{j}}\left(-\boldsymbol{V}_{\boldsymbol{j}}^{T} \boldsymbol{M}\left(\boldsymbol{p}, \omega_{j}\right) \boldsymbol{V}_{\boldsymbol{j}} \omega_{j}^{2}+j \boldsymbol{V}_{\boldsymbol{j}}^{T} \boldsymbol{C}\left(\boldsymbol{p}, \omega_{j}\right) \boldsymbol{V}_{\boldsymbol{j}} \omega_{j}+\boldsymbol{V}_{\boldsymbol{j}}^{T} \boldsymbol{K}\left(\boldsymbol{p}, \omega_{j}\right) \boldsymbol{V}_{\boldsymbol{j}}\right)^{-1} \boldsymbol{B}$

This approximation consists of a set of sampled key frequencies $\omega_{s}$ and corresponding interpolation matrices $\boldsymbol{W}_{\boldsymbol{i} j}$ which allow to evaluate the response matrix at frequency $\omega_{i}$ from the responses at $\omega_{j}$. At each of these frequencies $\omega_{j}$, the local response matrix is computed through a local reduced order basis (IROB) $\boldsymbol{V}_{\boldsymbol{j}}$.

\section{Reduced Order Model Setup}

The ROM setup as proposed in this work entails several steps:

- $\quad$ Parameter sampling to provide reference data for the ROM construction. This leads to a range of $n_{t r}$ training parameter sets $\boldsymbol{p}_{k}$ At these samples, the response matrices $\boldsymbol{H}\left(\boldsymbol{p}_{\boldsymbol{k}}, \boldsymbol{\omega}\right)$ are computed.

- The parametric dominant frequency sampling $\omega_{s}$ is performed on this data through a greedy-sampling procedure to obtain a sparse approximation:

$$
\begin{aligned}
& \min _{\boldsymbol{\omega}_{s}, \boldsymbol{W}}\left|\boldsymbol{\omega}_{\boldsymbol{s}}\right|_{0} \\
& \text { s.t }\left|\boldsymbol{H}\left(\boldsymbol{p}_{\boldsymbol{k}}, \boldsymbol{\omega}\right)-\boldsymbol{W H}\left(\boldsymbol{p}_{\boldsymbol{k}}, \boldsymbol{\omega}_{\boldsymbol{s}}\right)\right|_{2} \leq \mathrm{tol}
\end{aligned}
$$

The $L_{0}$ norm is approximated through a greedy sampling approach, and a user defined tolerance determines the stop criterion.

- $\quad$ For each of the retained frequency samples, a local reduced order basis is set up. A each frequency line, the responses are concatenated.

$$
\overline{\boldsymbol{H}}_{\boldsymbol{i}}=\left[\begin{array}{llll}
\boldsymbol{H}\left(\boldsymbol{p}_{\mathbf{1}}, \omega_{i}\right) & \ldots & \boldsymbol{H}\left(\boldsymbol{p}_{\boldsymbol{n}_{\boldsymbol{t r}}}, \omega_{i}\right)
\end{array}\right], \quad \forall \omega_{i} \in \boldsymbol{\omega}_{\boldsymbol{s}}
$$

singular value decomposition retaining only the $m$ dominant contributions:

$$
\begin{aligned}
& {[\boldsymbol{U}, \boldsymbol{\Sigma}, \boldsymbol{Q}]=\boldsymbol{\operatorname { v v d }}\left(\overline{\boldsymbol{H}}_{\boldsymbol{i}}\right)} \\
& \boldsymbol{V}_{\boldsymbol{i}}=\left\{\boldsymbol{U}_{\boldsymbol{j}} \mid \sigma_{j}>\sigma_{l}, j \leq m, l>m\right\}
\end{aligned}
$$

- $\quad$ Based on the samples and IROBs, the interpolation matrices $\boldsymbol{W}_{i j}$ are computed from a least squares approximation with respect to the training configurations.

The data which is stored for the reduced order model are therefore: frequency sampling points $\boldsymbol{\omega}_{s}$, local reduced order bases $\boldsymbol{V}_{\boldsymbol{i}}$ and interpolation matrices $\boldsymbol{W}_{\boldsymbol{i} \boldsymbol{j}}$.

\section{Reduced Order Model Evaluation}

For the online evaluation of the model, three main steps are followed: For a given parameter set $\boldsymbol{p}$, evaluate the (frequency dependent) model matrices $\boldsymbol{M}\left(\boldsymbol{p}, \omega_{s}\right), \mathbf{C}\left(\boldsymbol{p}, \omega_{s}\right)$ and $\boldsymbol{K}\left(\boldsymbol{p}, \omega_{s}\right)$ over the reduced frequency set. These matrices can be extracted from commercial software.

- $\quad$ Perform a Galerkin projection with the local reduced order bases for the corresponding frequencies.

- $\quad$ Evaluate the FRF according to the reduced Eq. (1).

\section{EXAMPLE}

The proposed space-frequency model order reduction scheme is demonstrated on a 500 DOF nominally homogenous mass-spring-damper model, as shown in Fig. 1.

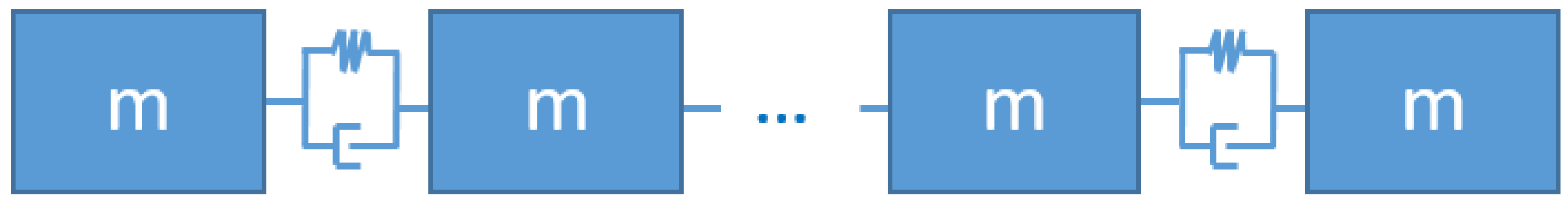

Figure 1: Mass-Spring-Damper model (500 masses)

For this system, the first two masses are parameterized and can vary between $5 \mathrm{~kg}$ and $10 \mathrm{~kg}$. The response obtained from the reduced model for a non-training parameter is shown in Fig. 2.

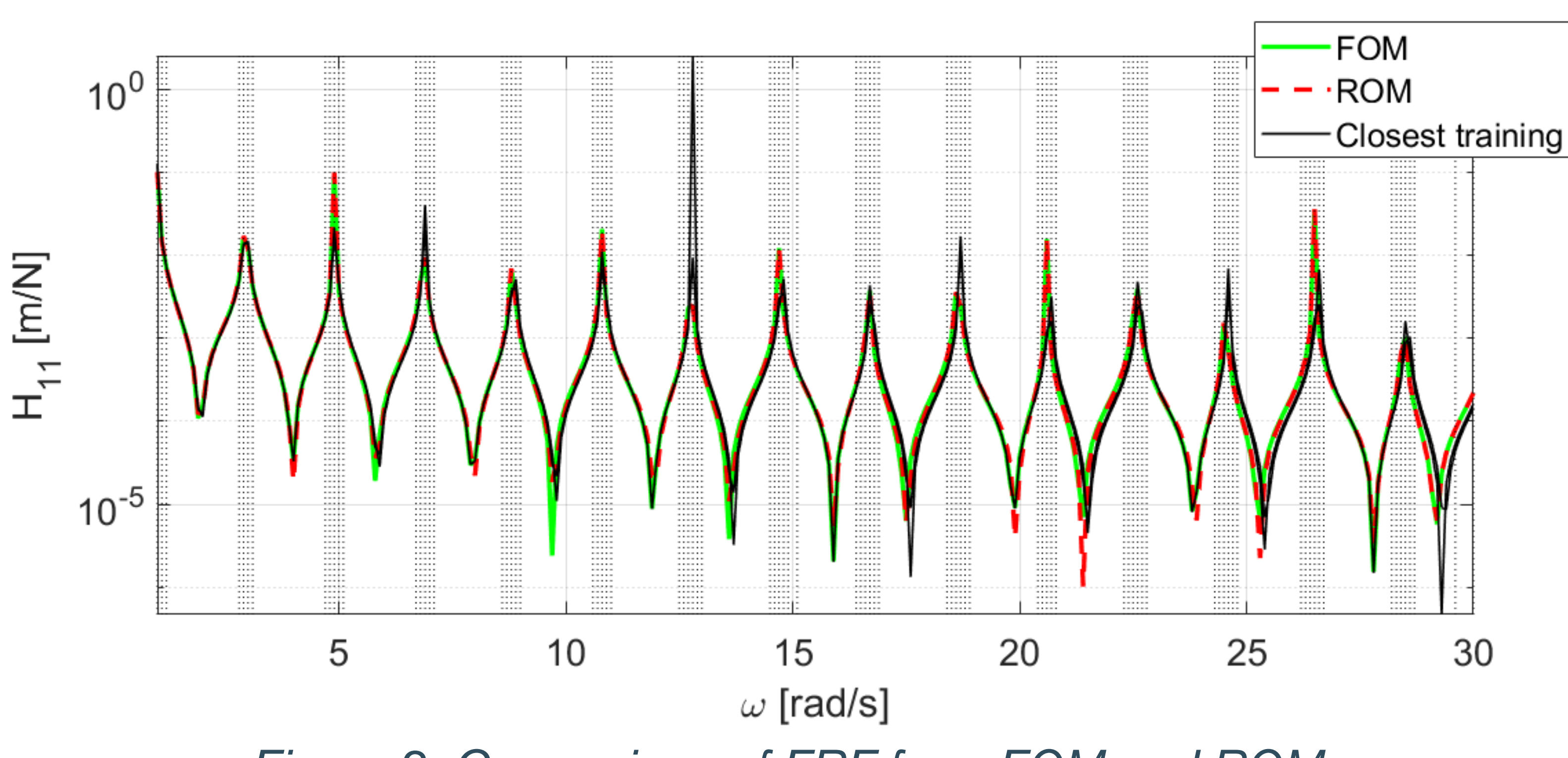

Figure 2: Comparison of FRF from FOM and ROM

For this example the reduced order model was only of order 5 at each of the 80 reduced frequency lines (vs 290 original frequency lines). This example demonstrates that the proposed method has the potential to considerably speed up multi-query parameterized frequency domain evaluations.

\section{HIGHLIGHTS}

- A pragmatic approach for parametric space-frequency reduction is proposed

- Method demonstrated on an academic case study show potential for the proposed approach

- $\quad$ Further research required on local ROB setup, parameter sampling and error indicators 